MS074.P11

\title{
Electroactive MOFs based on quinone/hydroquinone redox chemistry
}

Maka Vijay Kumar ${ }^{1}$, JaruguNarasimha Moorthy ${ }^{1}$

${ }^{1}$ Department Of Chemistry, Indian Institute Of Technology Kanpur, Kanpur, India

E-mail: makav@iitk.ac.in

The redox chemistry of quinones and hydroquinones is very important in biological systems. The therapeutic efficiency as well as toxicity of anthracycline anticancer drugs, a class of anthraquinones, is governed by their electrochemical properties. In general, quinones serve as important functional molecular components in diverse biological systems.

To exploit the redox chemistry of quinone/hydroquinone and develop electroactive functional MOFs, we have undertaken a systematic approach based on de novo design of organic linkers and their synthesis. The tetraacid TA shown in Figure- 1 is representative of some systems that we have begun to explore for metal-assisted self-assembly. We successfully synthesized some MOFs of the tetraacid with closed-shell metal ions such as $\mathrm{Zn} 2+$ and Cd2+. We are currently exploring redox properties of these MOFs. Results of these investigations will be presented.

[1] Lauren, E. K. et al. (2012). Chem. Rev.112, 1105-1125.

[2] Alessandro, D. M. D. et al. (2016). Chem. Commun. 52, 8957-8971.

[3] Dhakshinamoorthy, A. et al. (2015). Angew. Chem. Int. Ed. 55, 5414 -5445.

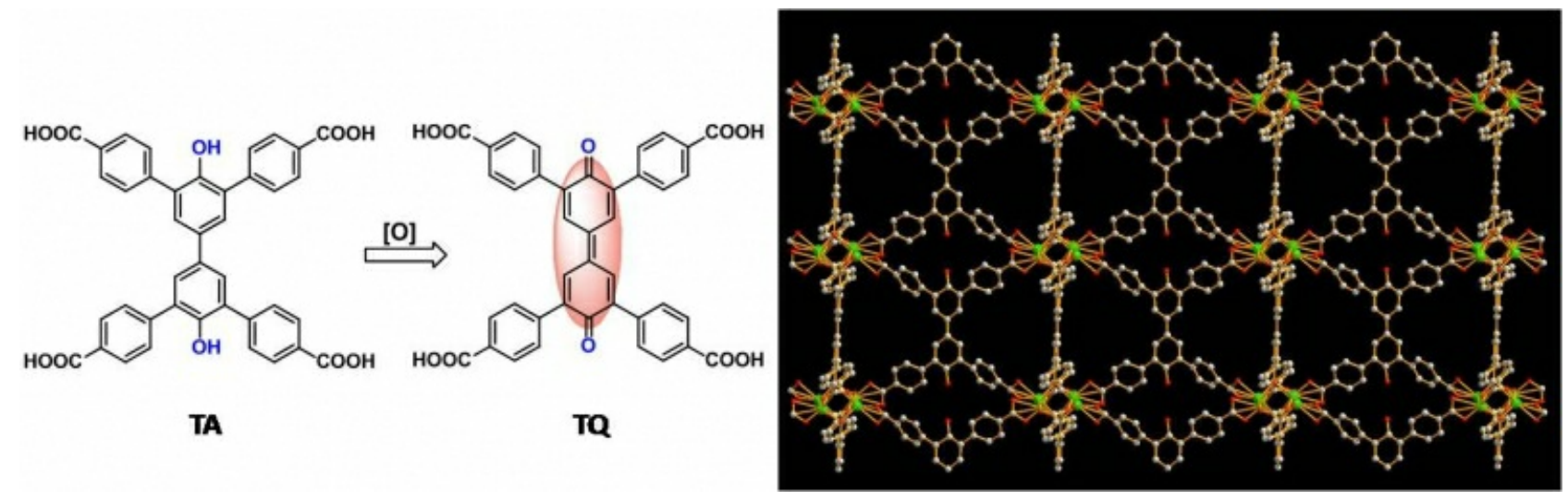

Keywords: Electroactive MOFs, Quinone/Hydroquinone Redox Chemistry, MOF 